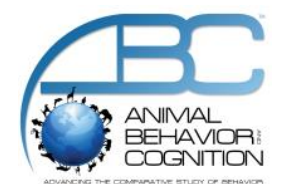

\title{
The Effect of Positive Reinforcement Training on an Adult Female Western Lowland Gorilla's (Gorilla gorilla gorilla) Rate of Abnormal and Aggressive Behavior
}

\author{
Austin Leeds ${ }^{1,2}$, Roby Elsner ${ }^{3}$, \& Kristen E. Lukas ${ }^{1,2}$ \\ ${ }^{1}$ Cleveland Metroparks Zoo \\ ${ }^{2}$ Case Western Reserve University \\ ${ }^{3}$ Audubon Zoo \\ *Corresponding author (Email: cal@ clevelandmetroparks.com)
}

Citation - Leeds, A., Elsner, R., \& Lukas, K. E. (2016). The effect of positive reinforcement training on an adult female Western lowland gorilla's (Gorilla gorilla gorilla) rate of abnormal and aggressive behavior. Animal Behavior and Cognition, 3(2), 78-87. doi: 10.12966/abc.02.05.2016

\begin{abstract}
Positive reinforcement training (PRT) has become a widely used tool in improving the ease with which husbandry and veterinary procedures are performed for animals under human care. PRT provides positive social interaction, cognitive stimulation, and choice, in addition to desensitization towards potentially stressful situations. As a result, PRT has been used as enrichment to decrease abnormal and aggressive behavior in various primate species, however, this has not been empirically tested in western lowland gorillas (Gorilla gorilla gorilla). This study used an ABA design to test the effect of PRT on the abnormal and aggressive behavior of an adult female gorilla both during and outside of interaction sessions. No change in behavior was observed during the PRT phase of this study. However, a decrease in ear covering and keeper-directed aggression were observed in the post-training period. Here we argue that the combination of both PRT and non-training interactions cumulatively provided social and cognitive stimuli resulting in the observed changes. These results provide further evidence on the importance of interactions between staff and animals in their care. Further systematic evaluation of the usefulness of PRT as enrichment is still needed, specifically in zoos and across different species. However, PRT is helpful in facilitating husbandry and veterinary procedures and thus should be considered an important tool in optimizing the welfare of animals under human care regardless of its effectiveness as enrichment.
\end{abstract}

Keywords - Abnormal behavior, Animal welfare, Human-animal interaction, Positive reinforcement training, Western lowland gorilla

The presence of stereotypic behavior, or repetitive and invariant behavior with no clear goal or function (Mason, 1991; Odberg, 1978), is commonly used as an indicator of poor welfare for animals in human care. The causes of stereotypic behavior have been attributed to an inability to perform speciestypical behavior, the presence of stimuli that are viewed as stressful, and/or being housed in an under stimulating environment (for review see Mason, 1991). The difficulty in using stereotypic behavior as a sign of suboptimal welfare is that stereotypic behavior can also be a "scar" of past experiences, can become habitual over time (Mason \& Latham, 2004; Mason \& Mendl, 1993), or may even be anticipatory of positive aspects of the animal's daily routine (Keen et al., 2014; Watters, 2014). Animals in human care also exhibit non-stereotypic behaviors, such as self-directed behaviors and undesirable behaviors that 
are considered signs of environmental deficiencies. Self-directed behaviors include a wide range of behavior from hair plucking (Less, Kuhar, \& Lukas, 2013) to self-injurious behavior (Hosey \& Skyner, 2007). Self-injurious behavior resulting in bodily harm represents a serious animal welfare concern and though the causes of these behaviors are not entirely understood, there is evidence that they may be a coping mechanism for stress (Novak, 2003). Undesirable behaviors are described as such because animals engage in them more frequently under human care than in the wild, they can be unsightly to visitors/staff, they may undermine the educational value of observing live animal behavior and/or are possibly maladaptive (for a more detailed review and discussion, see Lukas, 1999a). Such behaviors include regurgitation and reingestion $(\mathrm{R} / \mathrm{R})$, or the voluntary, retrograde movement of food or fluid from the esophagus or stomach into the mouth, a behavior observed frequently in western lowland gorillas (Gorilla gorilla gorilla) managed in zoos but never reported in wild populations (Lukas, 1999a). R/R is thought to be an adaptive response to deficiencies in diet and foraging opportunities and though the effect of $\mathrm{R} / \mathrm{R}$ on gorilla health is in need of more empirical investigation, similar behavior in humans can cause damage to the esophagus and result in other health complications (Tack, Blondeau, Boecxstaens, \& Rommel, 2011). In this article we will refer to stereotypic, self-directed and undesirable behaviors collectively as abnormal behavior for ease of discussion, though it should be noted that defining all forms of these behavior as "abnormal" is inappropriate (for review and discussion, which is out of the scope of this paper, see Lukas, 1999a; Mason, 1991; Zeller, 1991).

Positive reinforcement training (PRT), which consists of reinforcing animals with rewards for exhibiting desired behavioral responses, has become a prominent husbandry technique that increases the efficiency of husbandry and veterinary procedures (Bloomsmith, Stone, \& Laule, 1998; Bloomsmith et al., 2003; Broder, MacFadden, Cosens, Rosenstein, \& Harrison, 2008; Gresswell \& Goodman, 2011). PRT may provide the participating animal with positive social interaction, cognitive stimulation, and choice (Laule, Bloomsmith, \& Schapiro, 2003; Laule \& Whittaker, 2007). Due to these functions, PRT has been evaluated for its effectiveness in improving animal welfare. Baker et al. (2009) summarized two main approaches of PRT: "targeted training intervention" and "training as enrichment." Targeted training is individualized with the goal of eliminating a specific abnormal behavior and/or the cause of the behavior. Training as enrichment is similar to traditional environmental enrichment in that PRT is provided with the goal of providing general stimulation that may cause a change of behavior.

Several studies have evaluated the effect of PRT as a targeted intervention to reduce or eliminate abnormal behaviors in primates. In zoos, targeted training has reduced the stereotypic self-slapping of a female orangutan (Pongo pygmaeus, Raper, Bloomsmith, Stone, \& Mayo, 2002), and copraphagy and self-biting in a group of drills (Mandrillus leucophaeus, Cox, 1987; Desmond, Laule, \& McNary, 1987). Morgan, Howell, and Fritz (1993) provided targeted training to a male chimpanzee (Pan troglodytes) in a laboratory who frequently engaged in R/R. Following the intervention, R/R was decreased by half. Targeted training was also successful in reducing the self-injurious behavior of an adult male chimpanzee in a laboratory, in combination with environmental enrichment and pharmacological treatment (Bourgeois, Vazquez, \& Brasky, 2007).

Studies of PRT as enrichment in primates have revealed its effectiveness in reducing abnormal behaviors. Pomerantz and Terkel (2009) implemented PRT as enrichment with a group of chimpanzees in a zoo. Compared to baseline conditions, a significant decrease in on-exhibit abnormal and stress-related behaviors occurred after PRT sessions compared to baseline. In a laboratory, Baker et al. (2009) implemented training as enrichment for over 60 singly housed rhesus macaques (Macaca mulatta). Decreased frequencies of abnormal behavior were only observed in those that had the highest initial levels of abnormal behavior and the behavior still remained quite high. Bourgeois and Brent (2005) tested the effect of training as enrichment, social enrichment, food enrichment and non-food enrichment on 7 singly housed male olive baboons (Papio hamadryas anubis) selected for study based on their high levels of abnormal behavior. PRT significantly reduced the duration of all abnormal behaviors but social enrichment, which consisted of placing the baboons in social pairs within a larger enclosure, reduced the mean duration of behaviors more than PRT. In addition, Coleman and Maier (2010) compared rates of abnormal behavior between 6 singly housed female rhesus macaques receiving PRT to 5 singly housed 
female rhesus macaques not receiving PRT. After one month, the individuals receiving PRT had significantly reduced rates of abnormal behavior compared to the control individuals. Beyond the primate order, pacing was significantly reduced in African wild dogs (Lycaon pictus) following PRT sessions designed to facilitate veterinary procedures (Shyne \& Block, 2010).

Although unrelated to abnormal behavior, PRT as enrichment has also been documented to have additional benefits to animals under human care. Savastano, Hanson, and McCann (2003) implemented a PRT program with 86 individuals of 17 species of New World primates in a zoo. Although this report did not conduct systematic evaluation of the animals' behavior beyond the goals of training, it was qualitatively noted that following one year of PRT the animals no longer fled or aggressed upon animal care staff when they entered enclosures.

Western lowland gorillas (Gorilla gorilla gorilla) are a flagship species in zoos and frequently participate in PRT programs, but limited data exist on the effect of PRT on gorilla behavior. Carrasco et al. (2009) introduced combination informal play and structured training sessions to two female gorillas in a zoo to test if keeper-animal interactions had any effect on gorilla behavior. When compared to baseline, a decrease in on-exhibit stereotypic behavior was observed. Pizzutto, Nichi, Corrêa, Ades, and Alcindo (2007) similarly provided back-to-back PRT and informal social interaction sessions to a singly housed male gorilla and observed a reduction in abnormal behaviors. Although these results demonstrate positive findings for gorillas, neither study examined the effect of PRT independent of other intervention strategies. In addition, both studies only examined behavior following training sessions with no description of the effect on behavior during interaction sessions.

The purpose of this study was to examine the effect of PRT as enrichment on the behavior of a western lowland gorilla using an ABA design. The study subject was an adult female gorilla who frequently engaged in R/R, ear covering, and hand waving. This study examined the effect of PRT as enrichment on these behaviors both off exhibit during interactions with animal care staff and on exhibit outside of interactions. In addition, the subject had a history of keeper-directed aggression. This study further evaluated the effect of PRT as enrichment on keeper-directed aggression during interaction sessions.

\section{Method}

\section{Subject and Housing}

The study subject was a 30-yr-old adult female western lowland gorilla housed at Lincoln Park Zoo (LPZ) in Chicago, IL, USA (for exhibit details see Ross \& Lukas, 2006). The subject was born at LPZ in 1970 and removed for hand-rearing at 30 days, introduced to a conspecific surrogate in 1972, and integrated with a social group in 1973. Prior to the start of this study the subject was transferred between groups at LPZ due to social incompatibility. During this study she was housed with her adult female offspring (age 22), a wild caught female (age 42), and a blackback male (age 11). Unlike the subject's previous group, members of the new group participated in daily PRT sessions. The subject's participation in these sessions was postponed until this study was finalized and approved.

\section{Procedure}

Data were collected from February to June 2001, using an ABA design in 5-week intervals (Saudargas \& Drummer, 1996). Baseline interactions (A) consisted of hand feeding the subject preferred produce items from her usual diet in 5 min sessions. The subject was fed by hand frequently prior to this study, but the frequency and scheduling of hand feeding increased in her new group, providing a baseline condition novel to any previous experiences (from here on, hand feeding will be referred to as nontraining interactions). Treatment interactions (B) consisted of PRT in $5 \mathrm{~min}$ sessions, including the establishment of the bridge, an arbitrary signal such as the verbal command "OK" that is paired with a primary reinforcement such as food that over time becomes a reinforcer for desired behavior, followed by 
target training and the presentation of various body parts (for details on PRT see Laule \& Whittaker, 2007; Laule et al., 2003). Successful execution of targeted behaviors resulted in a reward of preferred produce items from her usual diet, which remained consistent throughout the entire study. During this study the subject was trained to target a PVC pipe and present her shoulder and was beginning to be shaped for tongue presentation. The subject was separated from her group during all sessions in an offexhibit holding area and freely participated. Voluntarily separating from her group was a normal aspect of her daily routine prior to the start of this study.

\section{Data Collection}

Baseline non-training interactions and PRT sessions occurred daily, but due to staffing limitations data were not collected during every session. To minimize variation, one keeper (RE) conducted the nontraining interactions and PRT while another keeper collected data. Non-training interactions and PRT were conducted between 0800 and 1000 and 1400 and 1500 in the off exhibit holding area. Data were collected during both the morning and afternoon sessions on days RE worked. Data were collected in 5min focal sessions (Altmann, 1974). All occurrences of abnormal and aggressive behaviors were recorded (see Table 1 for ethogram). Observations began at the beginning of the interaction session and ended after 5 min. For consistency with baseline conditions, PRT sessions were terminated at the 5-min mark. A total of 115 sessions were observed $(9.58 \mathrm{hrs})$. Across both baseline conditions 78 observations were conducted, and 37 observations were conducted in the PRT condition. On-exhibit data were collected by RE between 1000 and 1400 and 1500 and 1700 during 15-min focal sessions. All occurrences of abnormal behavior were recorded. These sessions were randomized and balanced evenly across time blocks. This design was selected to account for potential long acting effects of PRT on subject behavior. Future studies should consider conducting observations both immediately and several hours after sessions to account for both short- and long-term effects. A total of 125 on-exhibit observations were collected (31.25 hrs). Across both baseline conditions a total of 84 observations were conducted and 41 observations were conducted during the PRT phase.

Table 1

\begin{tabular}{ll}
\begin{tabular}{l} 
Ethogram of the Subject's Abnormal and Keeper-Directed Aggressive Behavior \\
\hline Behavior
\end{tabular} & Description \\
\hline $\begin{array}{l}\text { Abnormal Behavior } \\
\text { Ear-covering }\end{array}$ & $\begin{array}{l}\text { The subject places one or both hands over her ear(s). } \\
\text { The subject bends one or both of her hands at the wrist and } \\
\text { Hand-waving }\end{array}$ \\
$\begin{array}{ll}\text { swings the hand(s) side to side. } \\
\text { The voluntary retrograde movement of food and/or fluid from } \\
\text { the esophagus or stomach by the subject into the mouth, } \\
\text { hand(s), or a substrate, followed by the subsequent } \\
\text { consumption of the regurgitant (Lukas, 1999a). }\end{array}$ \\
$\begin{array}{ll}\text { Keeper-directed Aggression } \\
\text { Lunge }\end{array}$ \\
$\begin{array}{l}\text { The subject quickly thrusts her body towards animal care staff. } \\
\text { Grab }\end{array}$ & $\begin{array}{l}\text { The subject quickly attempts to reach out and grasp animal } \\
\text { care staff. }\end{array}$ \\
Bark & A sharp explosive vocalization towards animal care staff.
\end{tabular}

\section{Data Analysis}

Data from each phase were pooled and averaged to generate a mean rate of occurrence for each behavior. Results are presented as the mean \pm the standard error of the mean. Due to the single subject design all data were analyzed using Kruskall-Wallace tests to compare means across conditions. Mann- 
Whitney tests were used for post hoc analyses. A Bonferroni correction was applied and all effects are reported at the 0.0167 level of significance. All tests were run on SPSS Version 20 (Chicago, IL, USA).

\section{Results}

\section{Behavior on Exhibit}

The rate of $\mathrm{R} / \mathrm{R}$ in the initial baseline $(\mu=6.5, S E=1.1)$, training condition $(\mu=3.97, S E=1.1)$, and second baseline ( $\mu=1.6, S E=1.3$ ) was not significantly different, $H(2)=2.956, p=0.228$. The rate of ear covering in the initial baseline $(\mu=1.1, S E=0.2)$, training condition $(\mu=2.1, S E=0.6)$, and second baseline $(\mu=0.51, S E=0.5)$ was not significantly different, $H(2)=4.653, p=0.098$. The rate of hand waving in the initial baseline $(\mu=0.8, S E=0.2)$, training condition $(\mu=1.1, S E=0.3)$, and second baseline $(\mu=0.7, S E=0.3$ ) was not significantly different, $H(2)=4.132, p=0.127$ (Figure 1a).

A)

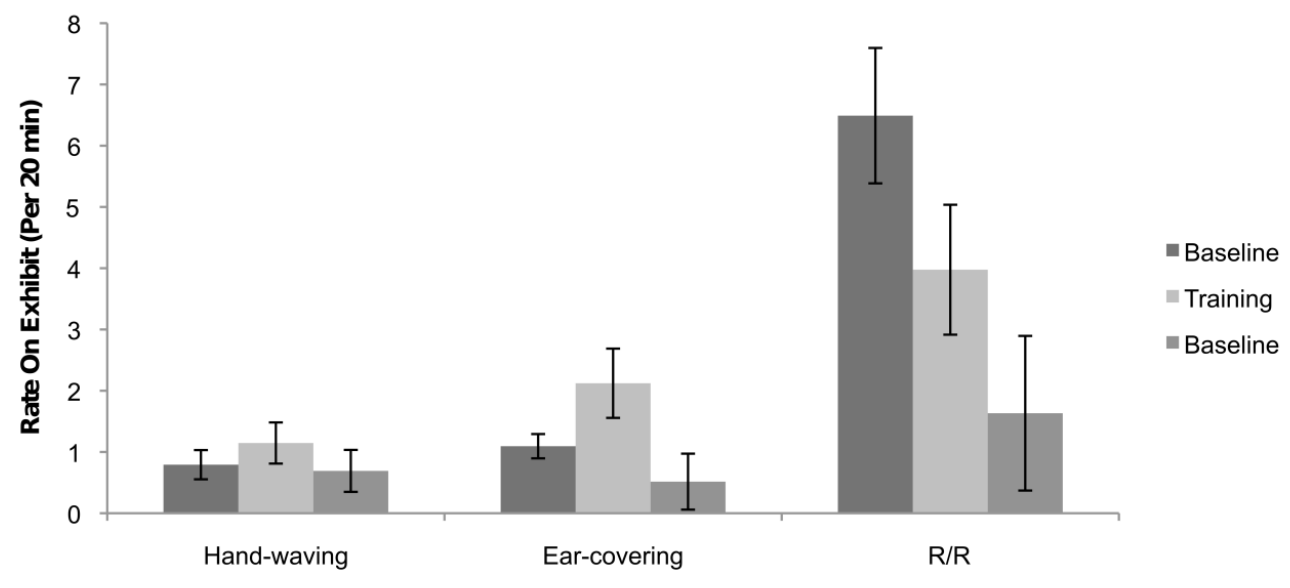

B)

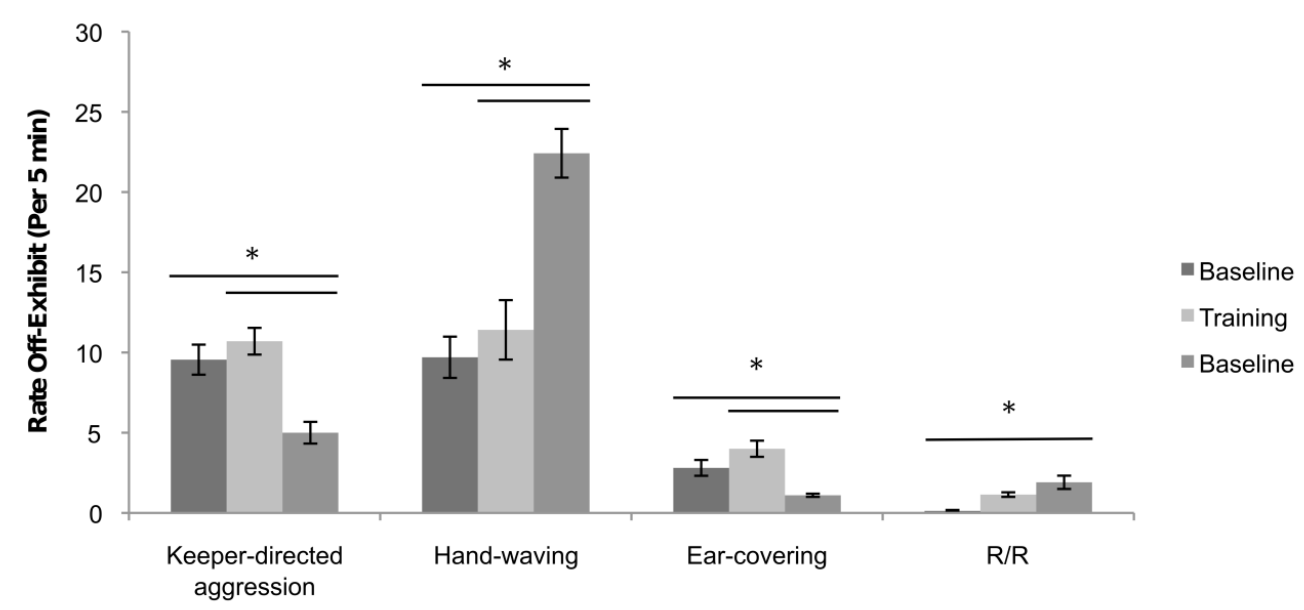

Figure 1. A) While on-exhibit no significant change in the rate of ear covering, hand waving or R/R was observed. B) During interaction sessions the subject's rate of ear-covering, $H(2)=27.91, p<0.001$, and keeper-directed aggression, $H(2)=20.36, p<$ 0.05 , significantly decreased in the second baseline. The subject's rate of hand-waving, $H(2)=30.75, p<0.001$, and $\mathrm{R} / \mathrm{R}, H(2)=$ $8.01, p=0.018$, however, significantly increased in the second baseline. 


\section{Behavior During Interactions}

The rate of keeper-directed aggression per session differed significantly, $H(2)=20.36, p<0.05$ across the initial baseline $(\mu=9.6, S E=0.9)$, training condition $(\mu=10.7, S E=0.8)$, and second baseline conditions $(\mu=5.0, S E=1.9)$. Post-hoc comparisons found that aggression was significantly lower in the second baseline compared to both the initial baseline, $U=406.5, z=-3.1, p=0.002, r=-0.373$, and training condition, $U=210.5, z=-4.5, p<0.001, r=-0.54$ (Figure 1B).

The rate of hand waving differed significantly, $H(2)=30.75, p<0.001$, across the initial baseline $(\mu=9.7, S E=1.29)$, training condition $(\mu=11.4, S E=1.85)$ and second baseline $(\mu=22.42, S E=1.52)$. Post hoc comparisons found that the rate of hand waving significantly increased in the second baseline compared to the initial baseline, $U=221.5, z=5.18, p<0.001, r=-0.59$, and training conditions, $U=$ 201.5, $z=-4.584, p<0.001, r=-0.56$ (Figure 1B).

The rate of ear covering differed significantly, $H(2)=27.91, p<0.001$, across the initial baseline $(\mu=2.81, S E=0.49)$, training condition $(\mu=4.0, S E=0.5)$, and second baseline $(\mu=1.1, S E=0.1)$. Post hoc comparisons revealed that the rate of ear covering was significantly lower in the second baseline compared to the training condition, $U=204.5, z=-4.767, p<0.001, r=-0.58$, and first baseline, $U=$ 240.0, $z=-5.19, p<0.001, r=0.59$ (Figure 1B).

The rate of $\mathrm{R} / \mathrm{R}$ differed significantly, $H(2)=8.01, p=0.018)$ across the initial baseline $(\mu=$ $0.13, S E=0.06)$, training condition $(\mu=1.14, S E=0.14)$ and second baseline $(\mu=1.91, S E=0.41)$. Post hoc comparisons found that the rate of $\mathrm{R} / \mathrm{R}$ significantly increased in the second baseline compared to the initial baseline, $U=539.5, z=-2.741, p=0.006, r=-0.31$ (Figure 1B).

\section{Discussion}

The purpose of this study was to evaluate if PRT as enrichment could be effective in reducing abnormal and aggressive behavior in an adult female western lowland gorilla. No significant change in the subject's abnormal behavior was observed during the PRT phase of this study, neither during training sessions nor outside of training sessions. It is interesting, however, that changes in behavior were observed in the second baseline during off-exhibit sessions. Due to the ABA design of this study, it is unclear if PRT as enrichment was effective in reducing the abnormal and aggressive behavior of this subject. A strict interpretation would say PRT had no effect, given that all observed changes occurred in the second baseline, indicating that non-training interactions (hand feeding) were associated with changes in behavior. However, if that were the case, we would expect to have observed similar levels of abnormal behavior in the initial baseline as well, which we did not. What differentiates the first and second baseline was the accumulation of PRT in the experimental phase. As a result, the two baselines are not truly identical in application. Here we will argue that the effects observed in the second baseline were the result of a carryover effect of PRT from the experimental phase and non-training interactions in the second baseline. We will additionally suggest that the subject's history was a confounding factor in this study and that more socially competent individuals may respond more readily to PRT.

In the zoo, interactions between staff and the animals in their care occur frequently and have the potential to provide both social and cognitive stimulation for the animals. Following an accumulation of twice-daily PRT and non-training interaction sessions with animal care staff, the subject of this study had a significant decrease in her rate of ear covering and keeper-directed aggression. Having consistent and positive interaction with animal care staff provides animals with predictability and choice in their environment, key contributors to optimizing animal welfare (for review, see Claxton, 2011). This subject likely benefited from such predictability and choice during interactions that also provided psychological stimuli, through the cognitive challenge of learning new behaviors, and social stimuli, by responding to verbal and visual cues of the animal care staff. In addition, physiological stimuli were provided by the physical challenges of training. As a result, ear covering, which has been described to occur following stressful events (Goerke, Fleming, \& Creel, 1987; Hardin, Danford, \& Skeldon, 1969; Woods, 2001), and 
keeper-directed aggression, which is often a sign of frustration or fear, decreased over time. Like more traditional environmental enrichment, PRT and non-training interactions provided the subject with stimuli that encouraged non-abnormal and non-aggressive behavior.

Although the goal of this study was to separately evaluate the impact of PRT as enrichment and non-training interactions, the results indicate that sequential implementation of both was effective treatment for abnormal and aggressive behavior in this gorilla. Similarly, Carrasco et al. (2009) and Pizzutto et al. (2007) found benefits of providing both PRT and non-training interaction with animal care staff to gorillas, though in these studies the two interventions were provided to gorillas simultaneously. Although further evaluation of PRT as enrichment is warranted from a research perspective, zoo animals are unlikely to experience PRT as enrichment as the sole interaction opportunity with animal care staff. In the zoo, animals have variable forms of interactions with staff each day and it is therefore an additional positive outcome of this study that the benefits of non-training interactions have again been documented, especially for a subject who historically had a primarily antagonistic relationship with animal care staff and conspecifics.

A novel finding of this study was the evidence that the combination of PRT and non-training interactions had an effect on the subject's affective state. In the second baseline, concurrent with the decrease in ear covering and keeper-directed aggression, the rate of hand waving increased. Though no empirical evaluations or descriptions of hand waving in gorillas have been conducted, the behavior was viewed as abnormal by staff in this study, and ultimately a sign of stress. However, there is increasing evidence that abnormal behavior is not always indicative of compromised welfare. Keen et al. (2014) found a positive correlation between positive affective states, as measured through cognitive bias testing, and pacing, prior to testing in grizzly bears (Ursus arctos horribilis). The authors concluded that the positive affective state was likely due to the enriching benefits of testing which included working for food rewards, and social and cognitive stimulation. Watters (2014) additionally argued that behaviors that may have historically been considered abnormal may also be anticipatory of positive events and indicate a positive affective state. Willemsen-Swinkels, Buitelaar, Dekker, and van Engeland (1998) reported a similar finding in their examination of abnormal behavior and mood in children with developmental disorders. When distressed, the children primarily exhibited sensory-low behaviors, defined as a repetitive sensory input of low intensity. This included using one's body as a surface for repetitive touching, objectively similar to the use of ear covering by our study subject. When elated, the children primarily exhibited movement limb behaviors, defined as the repetitive movement of one or two body parts, which included a behavior described as hand-flapping, objectively similar to the hand waving used by the present study subject. Willemsen-Swinkels et al. (1998) provided some of the first evidence that the use of different abnormal behaviors can be mood dependent, which corroborates more recent studies linking abnormal behavior and positive experiences in animals in human care. The observed switch in the present study subject's hand waving behavior was likely indicative of a change in affective state, and when examined in the context of the other behavioral changes, likely a change to a more positive affective state. This is the first evidence in a gorilla that PRT and non-training interactions with animal care staff can positively influence an animal's affective state.

Despite the decreases in ear covering and keeper-directed aggression, no significant change in $\mathrm{R} / \mathrm{R}$ was observed, which may be the result of the origins of the behavior. $\mathrm{R} / \mathrm{R}$ is frequently cited as a response to an inadequate diet and/or foraging opportunities (Lukas, 1999a) and, as a result, various dietrelated interventions have been successful in reducing it (Less et al., 2014; Lukas et al., 1999; Ruempler, 1992). Morgan et al. (1993) implemented a targeted PRT program with a male chimpanzee in a laboratory who engaged in R/R. The behavior decreased immediately following PRT but had no carry over to nontraining days. Pizzutto et al. (2007) found that a combination of play and training sessions had a similarly positive short-term effect on the $\mathrm{R} / \mathrm{R}$ of a solitary male gorilla. The data to date demonstrates that diet may be the most effective means with which to treat R/R, though few evaluations of other intervention strategies have been conducted. It should be noted, however, that though not statistically significant, there was a visual trend for a decrease in on-exhibit $\mathrm{R} / \mathrm{R}$ across conditions in the present study. It is possible that PRT as enrichment has value in reducing $R / R$, though further evaluation is needed. 
A surprising outcome of this study was the study subject's initial reaction to these PRT and nontraining interactions. In the initial two stages, the subject's rate of ear covering and keeper-directed aggression was approximately once and twice per minute, respectively. Although her rate of R/R during off-exhibit keeper interactions was almost zero during baseline, she exhibited $\mathrm{R} / \mathrm{R}$ at a rate of 18 times per $\mathrm{hr}$ on exhibit during baseline. This was consistent with reports she had the second highest rate of $\mathrm{R} / \mathrm{R}$ in a multi-institution study of gorilla R/R (Lukas, 1999b). When ear covering and keeper-directed aggression decreased off-exhibit in the second baseline, R/R increased. Given her history with R/R before this study, it can be argued that frequent $\mathrm{R} / \mathrm{R}$ was "normal" for her. The low rates in the initial off-exhibit phases, followed by an increase over time can then be described as acclimatization to a novel, and ultimately initially stressful, husbandry procedure. Given that even benign husbandry procedures can be stressful to animals in human care (Morgan \& Tromborg, 2007), the stress response to these sessions should not be surprising; however, no other study has documented such a response following the initiation of PRT and/or non-training interactions. This extended acclimatization period likely also accounts for the observed increases in the second baseline that were not observed in the first two phases. Although PRT and non-training interactions have been generally described as positive for the participating animal, this finding demonstrates the importance of designing PRT and non-training interaction plans for each specific individual based on their temperament and personal needs. Furthermore, this demonstrates the need of long-term studies on the effect of PRT as enrichment on animal welfare to properly account for any extended acclimatization periods that may occur.

Including this study, there are only two published studies on PRT as enrichment for primates in zoos. However, both zoo studies had conditions that may not be representative of most zoo primates. Pomerantz and Terkel (2009) reported a strong positive effect on the behavior of chimpanzees in a zoo following PRT. However, the subjects also spent $15 \mathrm{hrs}$ a day in holding areas, which were described as "fairly small and barren, and are not as complex and stimulating as the settings in other zoos may be." The present study involved a subject who was hand-reared, had a history of social incompatibility with conspecifics, and exhibited aggression toward animal care staff. Additionally, this study subject had a strong initial aversion to PRT and non-training interactions. From this it can be argued that the two evaluations of PRT as enrichment in zoos are not representative of the majority of zoo animals, specifically in accredited North American and European zoos. Further evaluations are needed to better understand how PRT as enrichment affects the behavior of animals in zoos. Specific focus should be placed on socially housed animals living in complex environments, preferably that had a species-typical upbringing. In addition, both zoo studies conducted training in off-exhibit areas during regularly scheduled times. Though many zoos conduct training sessions in this manner, it is not the industry standard. Many zoos conduct training randomly throughout the day, when time allows, and even conduct training across exhibit areas (i.e., outdoor exhibit, indoor day room, etc.). Evaluating how PRT affects the behavior of participants in these contexts also warrants further evaluation, as the less predictability may provide different stimuli to the participants.

This study provides further evidence on the value of regular and positive interactions between staff and animals in their care, PRT or otherwise. Further research is needed to improve our understanding of how PRT affects the behavior of animals under different management strategies, as well as understanding how PRT affects different taxa, specifically non-primates. Of particular focus should be long-term studies, as this study demonstrated that short-term evaluations may not be effective for subjects that require a longer familiarization period. Despite the lack of data on PRT as enrichment, PRT in general is helpful in facilitating husbandry and veterinary procedures and can simultaneously be used to maintain positive relationships between staff and animals, and thus should be considered an important tool in optimizing the welfare of animals under human care. 


\section{Acknowledgements}

The authors would like to thank the staff at Lincoln Park Zoo for their help in completing this research project. Additional thanks go to Bonnie Baird and Jason Wark for reviewing an early version of this manuscript.

\section{References}

Altmann, J. (1974). Observational study of behaviour: Sampling methods. Behaviour, 49, 227-267.

Baker, K. C., Bloomsmith, M., Neu, K., Griffis, C., Maloney, M., Oettinger, B., Schoof, V., \& Martinez, M. (2009). Positive reinforcement training moderates only high levels of abnormal behavior in singly housed rhesus macaques. Journal of Applied Animal Welfare Science, 12, 236-252.

Bloomsmith, M. A., Jones, M. L., Snyder, R. J., Singer, R. A., Gardner, W. A., Liu, S. C., \& Maple, T. L. (2003). Positive reinforcement training to elicit voluntary movement of two giant pandas throughout their enclosure. Zoo Biology, 22, 323-334.

Bloomsmith, M. A., Stone, A. M., \& Laule, G. E. (1998). Positive reinforcement training to enhance the voluntary movement of group-housed chimpanzees within their enclosures. Zoo Biology, 17, 333-341.

Bourgeois, S. R., \& Brent, L. (2005). Modifying the behaviour of singly caged baboons: Evaluating the effectiveness of four enrichment techniques. Animal Welfare, 14, 71-81.

Bourgeois, S. R., Vazquez, M., \& Brasky, K. (2007). Combination therapy reduces self-injurious behaviour in a chimpanzee (Pan troglodytes troglodytes): A case report. Journal of Applied Animal Welfare Science, 10, 123-140.

Broder, J. M., MacFadden, A. J., Cosens, L. M., Rosenstein, D. S., \& Harrison, T. M. (2008). Use of positive reinforcement conditioning to monitor pregnancy in an unanesthetized snow leopard (Uncia uncia) via transabdominal ultrasound. Zoo Biology, 27, 78-85.

Carrasco, L., Colell, M., Calvo, M., Abello, M. T., Velasco, M., \& Posada, S. (2009). Benefits of training/playing therapy in a group of captive lowland gorillas (Gorilla gorilla gorilla). Animal Welfare, 18, 9-19.

Claxton, A. (2011). The potential of the human-animal relationship as an environmental enrichment for the welfare of zoo-housed animals. Applied Animal Behaviour Science, 133, 1-10.

Coleman, K., \& Maier, A. (2010). The use of positive reinforcement training to reduce stereotypic behaviour in rhesus macaques. Applied Animal Behaviour Science, 124, 142-148.

Cox, C. (1987). Increase in the frequency of social interactions and the likelihood of reproduction among drills. Paper presented at the American Association of Zoological Parks and Aquariums Annual Conference, Portland, OR.

Desmond, T., Laule, G., \& McNary, J. (1987). Training for socialization and reproduction with drills. Paper presented at the American Association of Zoological Parks and Aquariums Annual Conference, Portland, OR.

Goerke, B., Fleming, L., \& Creel, M. (1987). Behavioural changes of a juvenile gorilla after a transfer to a more naturalistic environment. Zoo Biology, 6, 283-295.

Gresswell, C., \& Goodman, G. (2011). Case study: Training a chimpanzee (Pan troglodytes) to use a nebulizer to aid the treatment of airsacculitis. Zoo Biology, 30, 570-578.

Hardin, C. J., Danford, D., \& Skeldon, P. C. (1969). Notes on the successful breeding by incompatible gorillas, Gorilla gorilla at Toledo Zoo. International Zoo Yearbook, 9, 84-88.

Hosey, G., \& Skyner, L. (2007). Self injurious behavior in zoo primates. International Journal of Primatology, 28, 1431-1437.

Keen, H. A., Nelson, O. L., Robbins, C. T., Evans, M., Shepherdson, D. J., \& Newberry, R. C. (2014). Validation of a novel cognitive bias task based on difference in quantity of reinforcement for assessing environmental enrichment. Animal Cognition, 17, 529-541.

Laule, G., \& Whittaker, M. (2007). Enhancing nonhuman primate care and welfare through the use of positive reinforcement training. Journal of Applied Animal Welfare Science, 10, 31-38.

Laule, G. E., Bloomsmith, M. A., \& Schapiro, S. J. (2003). The use of positive reinforcement training techniques to enhance the care, management, and welfare of primates in the laboratory. Journal of Applied Animal Welfare Science, 6, 163-173.

Less, E. H., Bergl, R., Ball, R., Dennis, P. M., Kuhar, C. W., Lavin, S. R., ...Lukas, K. E. (2014). Implementing a low-starch biscuit-free diet in zoo gorillas: The impact on behavior. Zoo Biology, 33, 63-73. 
Less, E. H., Kuhar, C. W., \& Lukas, K. E. (2013). Assessing the prevalence and characteristics of hair-plucking behaviour in captive western lowland gorillas (Gorilla gorilla gorilla). Animal Welfare, 22, 175-183.

Lukas, K. E. (1999a). A review of nutritional and motivational factors contributing to the performance of regurgitation and reingestion in captive lowland gorillas (Gorilla gorilla gorilla). Applied Animal Behaviour Science, 63, 237-249.

Lukas, K. E. (1999b). The role of feeding motivation and individual differences in the development and maintenance of regurgitation and reingestion $(R / R)$ in captive lowland gorillas (Gorilla gorilla gorilla). Unpublished doctoral dissertation, Georgia Institute of Technology, GA.

Lukas, K.E., Hamor, G., Bloomsmith, M.A., Horton, C.L., \& Maple, T. L. (1999). Removing milk from captive gorilla diets: The impact on regurgitation and reingestion $(\mathrm{R} / \mathrm{R})$ and other behaviors. Zoo Biology, 18, 515528.

Mason, G. J. (1991). Stereotypies: A critical review. Animal Behaviour, 41, 1015-1037.

Mason, G. J., \& Latham, N. R. (2004). Can't stop, won't stop: Is stereotypy a reliable animal welfare indicator? Animal Welfare, 13, 57-69.

Mason, G., \& Mendl, M. (1993). Why is there no simple way of measuring animal welfare? Animal Welfare, 2, 301319.

Morgan, L., Howell, S. M., \& Fritz, J. (1993). Regurgitation and reingestion in a captive chimpanze (Pan troglodytes). Lab Animal, 22, 42-45.

Morgan, K. N., \& Tromborg, C. T. (2007). Sources of stress in captivity. Applied Animal Behaviour Science, 102, 262-302.

Novak, M. (2003). Self-injurious behaviour in rhesus monkeys: New insights into it etiology, physiology, and treatment. American Journal of Primatology, 59, 3-19.

Odberg, F. (1978). Abnormal behaviors: stereotypies. Paper presented at the First World Congress on Ethology Applied to Zootecnics, Madrid, Spain.

Pizzutto, C. S., Nichi, M., Corrêa, S. H. R., Ades, C., \& Alcindo, M. (2007). Reduction of abnormal behavior in a gorilla (Gorilla gorilla gorilla) through social interaction with a human being. Laboratory Primate Newsletter, 46, 6-10.

Pomerantz, O., \& Terkel, J. (2009). Effects of positive reinforcement training techniques on the psychological welfare of zoo-housed chimpanzees (Pan troglodytes). American Journal of Primatology, 71, 687-695.

Raper, J. R., Bloomsmith, M. A., Stone, A., \& Mayo, L. (2002). Use of positive reinforcement training to decrease stereotypic behaviours in a pair of orang-utans (Pongo pygmaeus). American Journal of Primatology, 57, 70-71.

Ross, S. R., \& Lukas, K. E. (2006). Use of space in a non-naturalistic environment by chimpanzees (Pan troglodytes) and lowland gorillas (Gorilla gorilla gorilla). Applied Animal Behaviour Science, 96, 143-152.

Ruempler, U. (1992). The Cologne zoo diet for lowland gorillas (Gorilla gorilla gorilla) to eliminate regurgitation and reingestion. Internation Zoo Yearbook, 31, 225-229.

Saudargas, R. A., \& Drummer, L. C. (1996). Single subject (small N) research designs and zoo research. Zoo Biology, 15, 173-181.

Savastano. G., Hanson, A., \& McCann, C. (2003). The development of an operant conditioning training program for New World primates at the Bronx Zoo. Journal of Applied Animal Welfare Science, 6, 247-261.

Shyne, A. \& Block, M. (2010). The effects of husbandry training on stereotypic pacing in captive African wild dogs (Lycaon pictus). Journal of Applied Animal Welfare Science, 13, 56-65.

Tack, J., Blondeau, K., Boecxstaens, V., \& Rommel, N. (2011). Review article: The pathophysiology, differential diagnosis and management of rumination syndrome. Alimentary Pharmacology \& Therapeutics, 33, 782788.

Watters, J. V. (2014) Searching for behavioral indicators of welfare in zoos: Uncovering anticipatory behaviour. Zoo Biology, 33, 251-256.

Willemsen-Swinkels, S. H. N., Buitelaar, J. K., Dekker, M., \& van Engeland, H. (1998). Subtyping stereotypic behavior in children: The association between stereotypic behavior, mood, and heart rate. Journal of Autism and Developmental Disorders, 28, 547-557.

Woods, S. (2001). Stress-related ear covering by captive great apes: A second look. Paper presented at the The Apes: Challenges for the 21st Century, Brookfield, IL.

Zeller, A. C. (1991). Human response to primate deviance. Anthropologica, 33, 39-68. 